\title{
Posterior midline and ventral parietal activity is associated with retrieval success and encoding failure
}

\section{Sander M. Daselaar ${ }^{1}$, Steven E. Prince ${ }^{2,3}$, Nancy A. Dennis ${ }^{4}$, Scott M. Hayes ${ }^{2,3}$, Hongkeun Kim ${ }^{5}$ and Roberto Cabeza ${ }^{2,3}$}

' Swammerdam Institute for Life Sciences, Faculty of Science, University of Amsterdam, Amsterdam, The Netherlands

2 Center for Cognitive Neuroscience, Faculty of Science, Duke University, University of Amsterdam, Amsterdam, The Netherlands

${ }^{3}$ Department for Psychology and Neuroscience, Faculty of Science, Duke University, University of Amsterdam, Amsterdam, The Netherlands

${ }^{4}$ Department of Psychology, Pennsylvania State University, University Park, PA, USA

${ }^{5}$ Department of Rehabilitation Psychology, Daegu University, Gyeongbuk, South Korea

\section{Edited by:}

Silvia A. Bunge, University of California

Berkeley, USA

Reviewed by:

Marian Berryhill, University of

Pennsylvania, Temple University, USA

*Correspondence:

Sander M. Daselaar, Swammerdam Institute for life Sciences, University

of Amsterdam, Kruislaan 320,

Amsterdam 1098 SM, The

Netherlands.

e-mail:s.m.daselaar@uva.nl
The ventral part of lateral posterior parietal cortex (VPC) and the posterior midline region (PMR), including the posterior cingulate cortex and precuneus, tend to show deactivation during demanding cognitive tasks, and have been associated with the default mode of the brain. Interestingly, PMR and VPC activity has been associated with successful episodic retrieval but also with unsuccessful episodic encoding. However, the differential contributions of PMR and VPC to retrieval vs. encoding has never been demonstrated within-subjects and within the same experiment. Here, we directly tested the prediction that PMR and VPC activity should be associated with retrieval success but with encoding failure. Consistent with this prediction, we found across five different $\mathrm{AMRI}$ experiments that, during retrieval, activity in these regions is greater for hits than misses, whereas during encoding, it is greater for subsequent misses than hits. We also found that these regions overlap with the ones that show deactivations during conscious rest. Our findings further aid in clarifying the role of the default mode regions in learning and memory.

Keywords: episodic memory, encoding, retrieval, default mode network, fMRI

\section{INTRODUCTION}

Human brain imaging studies have revealed a network of brain regions including parietal, posterior midline and frontal regions that consistently show deactivation during active task conditions compared to passive rest conditions in a wide variety of experiments (Mazoyer et al., 2001; McKiernan et al., 2003; Shulman et al., 1997). According to an influential theory - the default mode hypothesis - this network is engaged in specific processes that normally occur during the conscious resting state (Raichle et al., 2001). The default mode hypothesis further asserts that the regions within this network are tonically active, but temporarily shut down when available resources are needed for efficient cognitive performance, giving rise to deactivation in these areas. The interest in the functional significance of the default network has recently been intensified by indications of deviations from normal default activity in various clinical populations, including patients with Alzheimer's dementia (Greicius et al., 2004; Lustig et al., 2003; Petrella et al., 2007; Rombouts et al., 2005), schizophrenia (Liang et al., 2006; Liu et al., 2006), and autism (Kennedy et al., 2006). Yet, despite all this interest, the specific cognitive processes that are mediated by the "default" network remain unclear.

In order to clarify the default processes, it is critical to outline when the regions within the default network are activated and when they are deactivated. Two prominent areas within this network are the posterior midline region (PMR) and the ventral parietal cortex (VPC). PMR includes the posterior cingulate cortex (roughly BAs $31 / 30 / 29$ ) and the precuneus (roughly medial BA 7).VPC refers to lateral posterior parietal cortex ventral to the intraparietal sulcus, including the supramarginal gyrus (roughly Brodmann Area - BA 40 ) and the angular gyrus (roughly BA 39).

Interestingly, activity in PMR and VPC has not only been associated with the default network, but also with retrieval of past events - or episodic retrieval. Event-related fMRI studies of episodic retrieval found that PMR and VPC show greater activity when previously encoded items are correctly classified as "old" (retrieval hit) than when they are incorrectly classified as "new" (retrieval miss) (Henson et al., 2005; Prince et al., 2005; Wagner et al., 2005; Weis et al., 2004). In view of these findings, it has been suggested that PMR and VPC are directly engaged in processes supporting successful episodic retrieval (Wagner et al., 2005).

In sharp contrast, fMRI studies of episodic encoding suggest a reverse relation between memory performance and activity in PMR and VPC. These studies found that PMR shows less activity for items that are subsequently remembered (encoding hit) than for those that are subsequently forgotten (encoding miss) (Daselaar et al., 2004a; Otten and Rugg, 2001; Wagner and Davachi, 2001). This finding has been attributed to processes detrimental for encoding during misses (Otten and Rugg, 2001; Wagner and Davachi, 2001) or to processes beneficial for encoding during hits (Daselaar et al., 2004a), but these hypotheses are not necessarily incompatible.

Taken together, the seemingly contradictory findings in retrieval and encoding studies suggest that successful retrieval is a component of the default mode of the brain, but successful encoding is 
not. Yet, the inverse contribution of PMR and VPC to episodic retrieval vs. encoding has never been demonstrated within-subjects and within the same experiment. To address these issues, we tested two predictions. First, we tested the prediction that PMR and VPC would show an "encoding/retrieval flip". That is, we expected that these regions would be more activated for hits than misses during retrieval but less activated for subsequent hits than misses during encoding. To ensure the generalizability of the findings, we tested this "encoding-retrieval flip" prediction in five different eventrelated fMRI experiments of episodic encoding and retrieval using faces (Exp. 1), scenes (Exp. 2), word pairs (Exp. 3), and single words (Exp. 4 and 5). Second, we tested the prediction that the PMR and $V P C$ regions showing the encoding-retrieval flip are part of the default network. To test this prediction, we separately assessed whether the PMR and VPC regions showing the encoding/retrieval flip in the different experiments tend to be deactivated during active task blocks as compared to passive rest blocks. To this end, we measured their mean activation levels during three separate fMRI experiments with passive rest and active task blocks, including blocks of faces, scenes, and single words.

\section{MATERIALS AND METHODS EXPERIMENTS 1 AND 2 + BLOCKED TASKS 1 AND 2: FACES AND SCENES}

Parts of these data have been published elsewhere (Prince et al., 2009).

\section{Participants}

Nineteen participants (10 females), all students at Duke University, with an average age of $22.7(\mathrm{SD}=4.1)$ years were scanned and paid for their participation. Data from three participants were excluded due to equipment malfunction and inadequate behavioral performance. Written informed consent was obtained for each participant, and the study met all criteria for approval of the Duke University Institutional Review Board.

\section{Stimulus materials}

The stimuli for the memory tasks consisted of 144 faces (Experiment 1) and 144 scenes (Experiment 2). The stimuli for the default tasks consisted of 90 faces (Blocked Task 1) and 90 scenes (Blocked Task 2 ). Face pictures were obtained, with permission from an online database $^{1}$ and represent the age spectrum from young adults to older adults as well as different racial groups (Minear and Park, 2004). Common indoor and outdoor scenes were obtained from an online database ${ }^{2}$.

\section{Procedures}

The memory task included three face (Experiment 1 ) and two scene runs (Experiment 2) for encoding and the same number of runs for retrieval. Run presentation order was fixed based on pilot testing designed to elicit equivalent performance across tasks. Trial timing and jitter durations during encoding were also determined by pilot testing in order to attain similar performance. Retrieval trials in all conditions were $3 \mathrm{~s}$ in duration, followed by a variable jitter ranging from 1500 to $2500 \mathrm{~ms}$ (mean jitter length was $2 \mathrm{~s}$, mean trial

${ }^{1}$ http://agingmind.cns.uiuc.edu/facedb

${ }^{2}$ http://www.corbis.com length was $5 \mathrm{~s}$ ). Participants were encouraged to respond within the allotted period. Responses were not included in the analyses when the response time exceeded this period. The total number of old study trials was 108, yielding a total of 108 encoding trials and 108 retrieval trials per condition.

For encoding trials, participants rated either the friendliness of a face (Experiment 1), or the pleasantness of a scene (Experiment 2) on a 1-4 scale (finger order was counterbalanced across participants) while learning the information (intentional encoding). For retrieval trials, participants made combined old/new confidence level decisions via four response choices (definitely old, probably old, probably new, definitely new).

Blocked Tasks 1 and 2 (Faces and Scenes) were performed after the memory tasks during a separate scan run. Subjects alternately viewed blocks of 15 faces (Blocked Task 1) or blocks of 15 scenes (Blocked Task 2) intermixed with 10-s rest blocks consisting of a fixation cross-hair. They were instructed to press a button when any of the stimuli repeated, which actually never occurred. Face and Scene stimuli were shown for $750 \mathrm{~ms}$ with a 250-ms gap. In total, there were six cycles (FACE-fixation-SCENE-fixation) and the start order was counterbalanced across subjects.

\section{EXPERIMENT 3: WORD PAIRS}

Parts of these data have been published elsewhere (Prince et al., 2005, 2007).

\section{Participants}

Fourteen participants (five female), all students at Duke University with an average age of $19.8(\mathrm{SD}=1.7)$ years, were scanned and paid for their participation. Written informed consent was obtained for each participant, and the study met all criteria for approval of the Duke University Institutional Review Board.

\section{Stimulus materials}

The critical stimuli consisted of 240 five-letter words that were selected from the MRC Psycholinguistic Database ${ }^{3}$. The words were of moderate frequency (mean 539), concreteness (mean 504), and imageability (mean 510).

\section{Procedures}

In total there were four experimental runs. Each run consisted of a study block and a recognition block separated by a 30-s delay. During the study blocks, two words in plain font were displayed next to each other in the center of the screen. Subjects were instructed to rate the semantic relatedness of the words on a 4-point scale ( $1=$ low; $4=$ high) and, at the same time, to try to memorize the word pair. Recognition memory was tested by presenting pairs identical to the ones studied and pairs made by recombining words from different studied pairs. Participants indicated whether each pair was identical or recombined and their level of confidence $(1=$ definitely identi$\mathrm{cal}, 2$ = probably identical, 3 = probably recombined, $4=$ definitely recombined). The trials were presented for $3.4 \mathrm{~s}$ and followed by an interval ranging between 0 and $5.4 \mathrm{~s}$.

Participants were encouraged to respond within the time that the word pair was displayed on the screen. Responses were not included

${ }^{3}$ http:/www.psy.uwa.edu.au/MRCDataBase/mrc2.html 
in the analyses when the response time exceeded this period. The number of identical trials per block was 29, and the number of recombined trials 16 yielding a total of 116 identical trials and 64 recombined trials.

\section{EXPERIMENT 4 + BLOCKED TASK 3: SINGLE WORDS Participants}

Nineteen Duke University students (seven female) with an average age of $22.86(\mathrm{SD}=2.72)$ years participated in the study. Written informed consent was obtained for each participant, and the study met all criteria for approval of the Duke University Institutional Review Board.

\section{Stimulus materials}

The critical stimuli consisted of 240 words that were selected from the MRC Psycholinguistic Database ${ }^{4}$. The words were of moderate familiarity (mean 78), and concreteness (mean 491).

\section{Procedures}

The memory task included four encoding runs, each with a duration of $392 \mathrm{~s}$ and six recognition runs, each with a duration of $392 \mathrm{~s}$. The encoding and retrieval phases were separated by a period of about 20 min during which high resolution anatomical and diffusion tensor images were acquired. During the first two encoding runs participants performed an animacy task and for the latter two were simply asked to read and remember the words for a later memory task. For the present study, these two encoding tasks were treated as one condition. During the recognition run, participants saw a mix of 240 old words shown during the encoding phase and 120 completely new words. They indicated whether the word was old or new, and how confident they were (definitely old, probably old, probably new, definitely new). During both memory phases, words were presented at a rate of $2.5 \mathrm{~s}$ per item followed by an interval ranging from $1-5 \mathrm{~s}$. Participants were encouraged to respond within the time that the word was displayed on the screen. Responses were not included in the analyses when the response time exceeded this period. For Blocked Task 3, we exploited the fact that the encoding and retrieval runs were designed as a mixed model, such that 60 -s task blocks (in which 12 words were presented per task block) alternated with 15-s passive rest blocks. During rest blocks participants simply viewed a fixation cross-hair and awaited the start of the next task block. For Blocked Task 3, only the word encoding blocks were included in the analysis.

\section{MEMORY EXPERIMENT 5: SINGLE WORDS \\ Participants}

Fourteen Duke University students (eight female) with an average age of $20.7(S D=2.3$ ) years participated in the study. Written informed consent was obtained for each participant, and the study met all criteria for approval of the Duke University Institutional Review Board.

\section{Stimulus materials}

The critical stimuli consisted of 250 words that were selected from the MRC Psycholinguistic Database ${ }^{5}$. The words were of moderate

${ }^{4}$ http:/www.psy.uwa.edu.au/MRCDataBase/mrc2.html

${ }^{5}$ http:/www.psy.uwa.edu.au/MRCDataBase/mrc2.html frequency (mean 528), concreteness (mean 553), and imageability (mean 552).

\section{Procedures}

The task included one encoding run with a duration of $429 \mathrm{~s}$ and one recognition run with a duration of $591 \mathrm{~s}$. The encoding and retrieval phases were separated by a period of about $45 \mathrm{~min}$ during which another task was performed. This task involved semantic ratings about word triplets, and will be reported elsewhere. During the encoding phase, participants studied a list of 100 words at a rate of 3 s per item while making semantic judgments about the words (manmade, artificial, or both?). They were told that there would be a later memory test for the words. During the recognition run, participants saw a mix of 100 old words shown during the encoding run and 50 completely new words. They indicated whether they were confident the word was old ("old"), whether they were confident the word was new ("new"), or whether they were not sure ("pass"). The trials were presented for $2 \mathrm{~s}$ and followed by an interval ranging between 0 and 4.5 s. Participants were encouraged to respond within the time that the word was displayed on the screen. Responses were not included in the analyses when the response time exceeded this period.

\section{fMRI SCANNING}

For the first three experiments (faces, scenes, word pairs) as well as the first two Blocked Tasks (faces, scenes), images were collected using a 4T GE scanner. High-resolution T1-weighted structural images were acquired with a 450-ms repetition time (TR), a 9-ms echo time (TE), a 24-cm field of view (FOV), a 2562 matrix, and a slice thickness of $1.9 \mathrm{~mm}$. Functional scanning employed an inverse spiral sequence with either a 1500-ms TR (experiments 1 and 2, and 4) or a 1700-ms TR (experiment 3), a 31-ms TE, a 24-cm FOV, a 642 image matrix, and a $60^{\circ}$ flip angle.

For the fourth experiment as well as Blocked Task 3 (single words), images were collected using a $3 \mathrm{~T}$ GE scanner. Highresolution T1-weighted structural imaging involved a $450-\mathrm{ms}$ repetition time (TR), a 3-ms echo time (TE), a $24-\mathrm{cm}$ field of view (FOV), a $256 \times 224$ matrix, and a slice thickness of $1.9 \mathrm{~mm}$. Functional scanning involved an inverse spiral sequence with a 2000-ms TR, a 27-ms TE, a 24-cm FOV, a 642 image matrix, and a $60^{\circ}$ flip angle.

For the fifth memory experiment (single words), images were collected using a 1.5T GE scanner. High-resolution T1-weighted structural imaging involved a 450-ms repetition time (TR), a 9-ms echo time (TE), a 24-cm field of view (FOV), a 2562 matrix, and a slice thickness of $1.9 \mathrm{~mm}$. Functional scanning involved an inverse spiral sequence with a $1500-\mathrm{ms}$ TR, a 31-ms TE, a 24-cm FOV, a 642 image matrix, and a $60^{\circ}$ flip angle.

For all five experiments, 34 contiguous slices were acquired with the same slice prescription as the anatomical images. Slice thickness was $3.75 \mathrm{~mm}$, resulting in cubic $3.75 \mathrm{~mm}^{3}$ isotropic voxels. Scanner noise was reduced with ear plugs and head motion was reduced with foam pads and headbands. Stimuli were presented with LCD goggles (Resonance Technology, Inc.), and behavioral responses recorded with a 4-key fiber-optic response box (Resonance Technology, Inc.). 


\section{fMRI ANALYSES}

Data from all five experiments as well as the three Blocked Tasks were analyzed using SPM2 (Statistical Parametric Mapping; Wellcome Department of Cognitive Neurology) ${ }^{6}$. Time-series were corrected for differences in slice acquisition times, and realigned. Functional images were spatially normalized to a standard stereotactic space, using the Montreal Neurological Institute (MNI) templates implemented in SPM2 and resliced to a resolution of $3.75 \times 3.75 \times 3.75 \mathrm{~mm}$. The coordinates were later converted to Talairach and Tournoux's space (Talairach and Tournoux, 1988). Finally, the volumes were spatially smoothed using an 8-mm isotropic Gaussian kernel and proportionally scaled to the whole-brain signal.

Trial-related activity (Exp. 1-5) was assessed by convolving a vector of the onset times of the stimuli with a synthetic hemodynamic response function (HRF). Block-related activity (Blocked Tasks 1-3) was assessed by convolving a boxcar function representing block onsets and offsets with the HRF. The general linear model, as implemented in SPM2, was used to model the effects of interest and other confounding effects (e.g., head movement and magnetic field drift). Statistical Parametric Maps were identified for each participant by applying linear contrasts to the parameter estimates (beta weights) for the events or blocks of interest, resulting in a $t$-statistic for every voxel.

For experiments 1-5, we coded four trial types: encoding hits, encoding misses, retrieval hits, and retrieval misses. Similar to other subsequent memory studies, only high-confidence retrieval hits were considered as hits and all other trials were modeled as retrieval miss (Otten et al., 2006; Schon et al., 2004). Experiment 5 (single words) did not include confidence ratings, but here participants were encouraged to use a "pass" option whenever they were not confident. Consequently, all hit responses were considered as retrieval hits here, while pass responses and misses were modeled as retrieval misses. Encoding hits and misses were determined by matching the retrieval hits and misses at test to the relevant trials at study.

In order to test the prediction that PMR and VPC would be more activated for retrieval hits than misses but less activated for encoding hits than misses in each of the five experiments, we used a four-step approach. First, using the Wake Forrest PickAtlas toolbox $^{7}$, we selected regions of interest (ROIs) in PMR comprising the precuneus and posterior cingulate (BA7/29/30/31) and VPC comprising the inferior parietal lobe, and supramarginal and angular gyri (BA39/40). Second, we identified regions within the ROIs showing a phase (encoding, retrieval) x success (hit, miss)

${ }^{6}$ http://www.fil.ion.ucl.ac.uk/spm

${ }^{7}$ http://www.fmri.wfubmc.edu interaction by calculating the contrasts retrieval hits $>$ misses, and encoding hits $<$ misses, both at an uncorrected threshold of $P<0.05$. Subsequently, we performed a conjunction analysis of these contrasts by inclusively masking the resulting $\mathrm{T}$ maps. As a third step, we plotted the mean cluster activity (parameter estimates) for $\mathrm{EH}, \mathrm{EM}, \mathrm{RH}$, and RM trials from the regions identified in the previous step.

In addition, in order to assess whether the regions that were identified in experiments $1-5$ tend to show significant deactivation during active task as compared to passive rest blocks, we extracted their mean activity during Blocked Tasks 1-3.

\section{RESULTS}

\section{BEHAVIORAL PERFORMANCE}

The hits - false alarm indices for Experiments $1-5$ were 0.52 , $0.49,0.37,0.46$, and 0.44 respectively, indicating good discrimination between old and new items in all five experiments. Response times are listed separately for encoding and retrieval phases in Table 1.

\section{fMRI RESULTS}

\section{Encoding-retrieval flip}

We predicted that, during encoding, activity in PMR and VPC would be greater for encoding misses than for hits, whereas during retrieval, activity would be greater for retrieval hits than misses. The results generally confirmed our predictions. As indicated in Figure 1, we found clusters of activity within PMR showing a negative success effect (miss $>$ hit) during encoding but a positive success effect (hit $>$ miss) during retrieval in all five experiments (maxima in Talairach $[x, y, z]$ based on retrieval hits $>$ misses contrast: Exp. 1: $[-12,-43,34]$, cluster size in voxels $(\mathrm{c})=71$; Exp. 2: $[11,-49,28], \mathrm{c}=192$; Exp. 3: [4, -50, 30], c = 146; Exp. 4: [8, -42, 37], $\mathrm{c}=5$; Exp. 5: $[-8,-42,33], \mathrm{c}=222)$.

As shown in Figure 2, we also found clusters of activity within VPC showing a negative success effect during encoding but a positive success effect during retrieval in all five experiments (Exp. 1: $[-40,-53,41], c=7 ;$ Exp. 2: [-54,-51,33], c = 18; Exp. 3: [-45, -54 , $31], c=4$; Exp. 4: $[-46,-54,29], c=6$; Exp. 5: $[-57,-46,23], c=5)$ and right (Exp. 1: [49,-59,34], c = 9; Exp. 2: [58,-51, 21], c = 65; Exp. 3: $[45,-54,34], c=23$; Exp. 4: [54, - 52, 29], c = 4; Exp. 5: $[56,-57,31], \mathrm{c}=18)$.

\section{Overlap of the flip pattern across all experiments}

Although the activations listed above all fell within PMR and VPC, we conducted an additional conjunction analysis to check the robustness of the locations across experiments. To this end, we calculated

Table 1 | Reaction times in $\mathbf{m s}$ (SD).

\begin{tabular}{|c|c|c|c|c|}
\hline & \multicolumn{2}{|c|}{ Encoding } & \multicolumn{2}{|c|}{ Retrieval } \\
\hline & Hits & Misses & Hits & Misses \\
\hline Exp. 2 Scenes & $1582(340)$ & $1527(271)$ & $1529(249)$ & 2194 (298) \\
\hline Exp. 3 Word pairs & $2088(233)$ & 2075 (343) & 1689 (198) & $1923(294)$ \\
\hline Exp. 4 Single words & $1234(144)$ & $1562(156)$ & $1485(366)$ & 1507 (399) \\
\hline
\end{tabular}




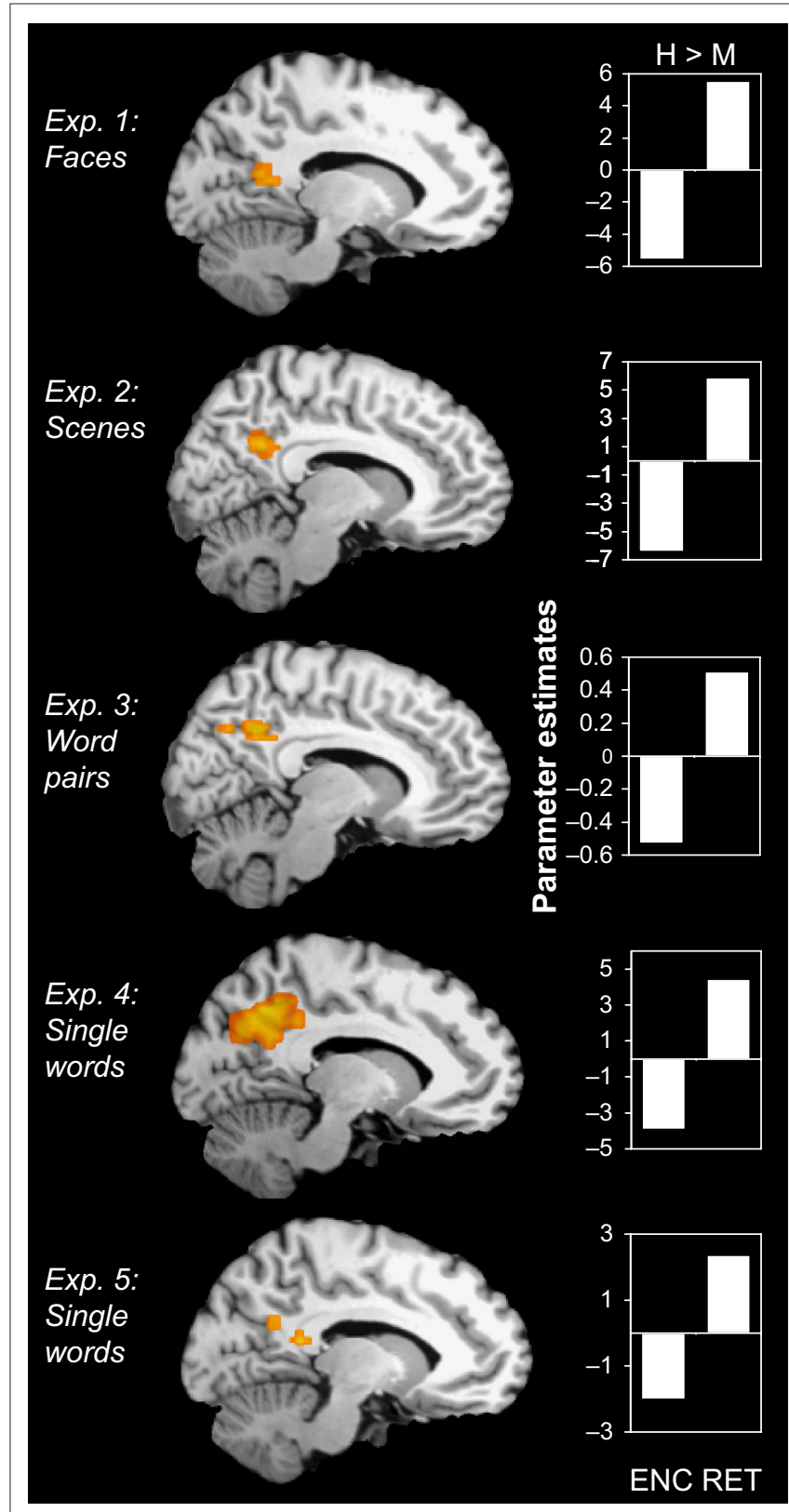

FIGURE 1 | Encoding/retrieval flip in PMR. Consistent results in five different $f M R I$ experiments: During encoding, activity in the posterior midline region (PMR) was greater for misses $(M)$ than for hits $(H)$, whereas during retrieval, activity was greater for hits than for misses. Bar graphs indicate mean cluster activity for the comparison between hits and misses during encoding and retrieval, respectively.

overlaps not only within experiments but also across all experiments. In other words, this conjunction analysis involved two T maps, one for encoding (miss > hit) and one for retrieval (hit > miss), for each of the five experiments yielding a total of 10 different $\mathrm{T}$ maps. The only region surviving this analysis was an area that fell within PMR (BA 31, Talairach $[x, y, z]=[-8,-45,37])$, and we did not find this perfect overlap within VPC areas. These findings indicate that compared to VPC, the encoding/retrieval flip pattern in PMR is more robust and less sensitive to specific task characteristics.

\section{Overlap with default network}

In addition to the encoding/retrieval flip, we predicted that PMR and VPC would show deactivation during active task as compared to passive rest blocks. As indicated by the bar graphs in Figure 3, the PMR and VPC regions identified in Exp. 1-5 all showed reliable task-induced deactivation during the three blocked fMRI tasks (Blocked Task 1: faces-F; Blocked Task 2: scenes-S; Blocked Task 3: words - W). As shown in Table 2, these observations were confirmed by one-sample $t$ tests of mean cluster activity relative to the passive resting baseline. There was only one region in one of the experiments that did not show a significant deactivation for all three blocked fMRI tasks, namely the left VPC region identified in Exp 1. We also assessed whether the use of proportional scaling in the fMRI analyses artificially yielded deactivations relative to the resting baseline. Yet, as shown in Figure 1 of Supplementary Material, the same analysis without scaling yielded almost identical results. Thus, in general, our results indicate that the PMR and VPC regions that show the encoding/retrieval flip are overlapping with the ones that are commonly deactivated during active task performance and that have been associated with the default mode of the brain.

\section{CONCLUSION AND DISCUSSION}

The goal of the present study was to investigate the differential role of PMR and VPC in successful episodic encoding vs. retrieval. The results confirmed our two predictions. First, in five different fMRI experiments using different stimuli, we confirmed the predicted encoding-retrieval flip activation pattern in PMR and VPC regions. Within the same participants, activity in these regions was associated with successful retrieval (retrieval hits $>$ misses) but with unsuccessful encoding (encoding misses $>$ hits). Second, we confirmed the prediction that encoding-retrieval flip regions overlap with the default network. All but one of the regions identified showed significant deactivation during three different active task blocks compared to rest. Below, we discuss this finding in relation to the default mode network, theoretical implications of the encoding/retrieval flip, and the role of VPC in retrieval.

\section{RELATION TO DEFAULT MODE NETWORK}

PMR and VPC consistently show deactivation during the performance of demanding cognitive tasks, presumably because they are part of a default mode that needs to be suppressed during efficient cognitive processing (Gusnard and Raichle, 2001). The processes that are mediated by the default network are still unclear, but can be clarified by outlining when default regions are activated and when they are deactivated. In five different fMRI experiments using different stimuli, we consistently found that both PMR and VPC show reduced activity during successful encoding but increased activity during successful retrieval.

The finding that PMR and VPC consistently show less activity for items that are successfully encoded (encoding hits) than for those that are later forgotten (encoding misses) replicates previous fMRI studies of episodic encoding (Daselaar et al., 2004a; Kao et al., 2005; Miller et al., 2008; Otten and Rugg, 2001; Shrager et al., 2008; Wagner and Davachi, 2001). Several of these studies (Daselaar et al., 2004a; Kao et al., 2005; Miller et al., 2008; Shrager et al., 2008) found that this effect actually reflects greater deactivations for encoding hits than misses trials. Based on the finding that both PMR and VPC 


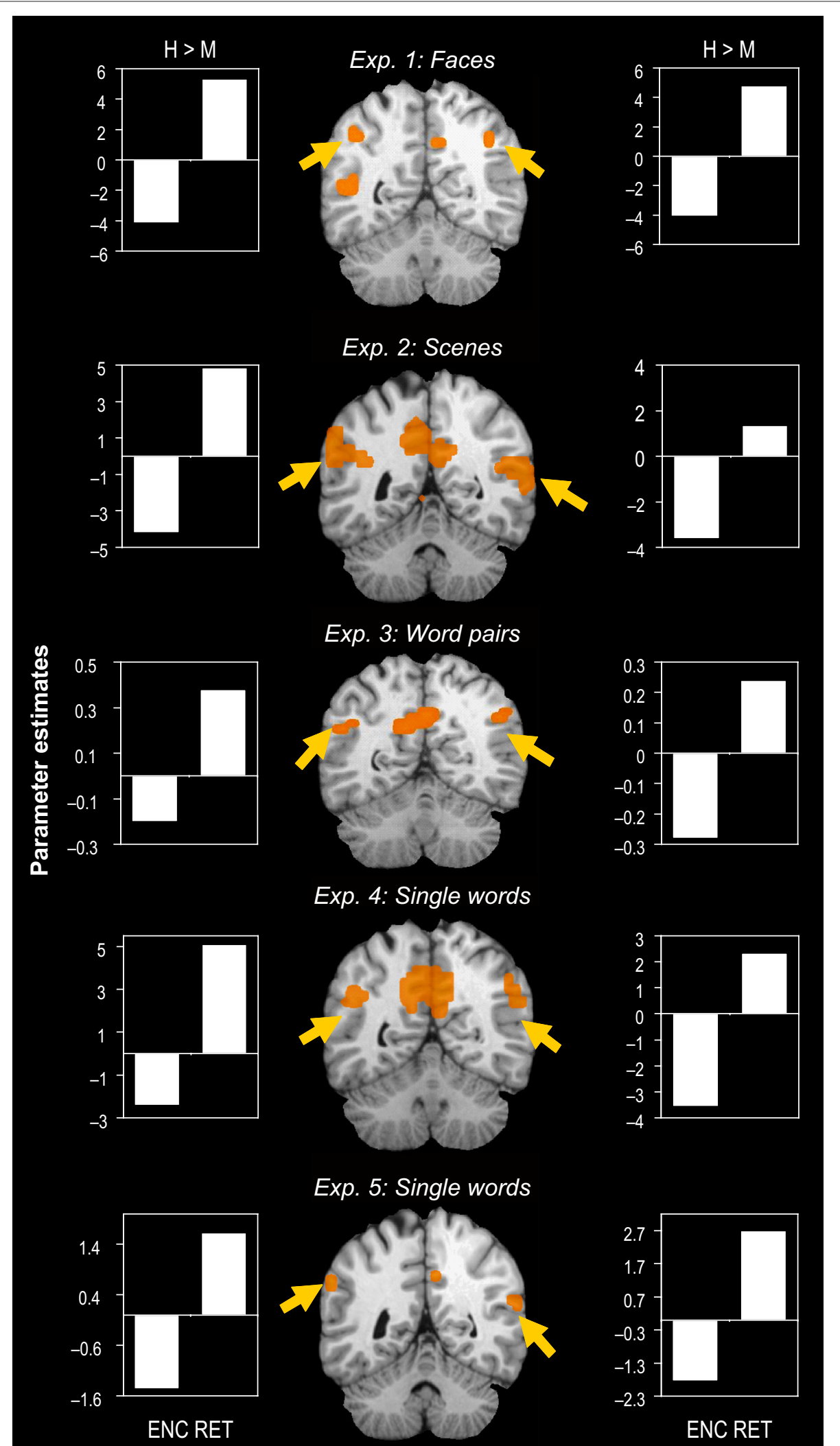

FIGURE 2 | Encoding/retrieval flip in VPC. Consistent results in five different $\mathrm{fMRI}$ experiments: During encoding, activity in both left and right ventral parietal cortex (VPC) was greater for misses (M) than for hits $(\mathrm{H})$, whereas during retrieval, activity was greater for hitss than for misses. Bar graphs indicate mean cluster activity for the comparison between hits and misses during encoding and retrieval, respectively. 


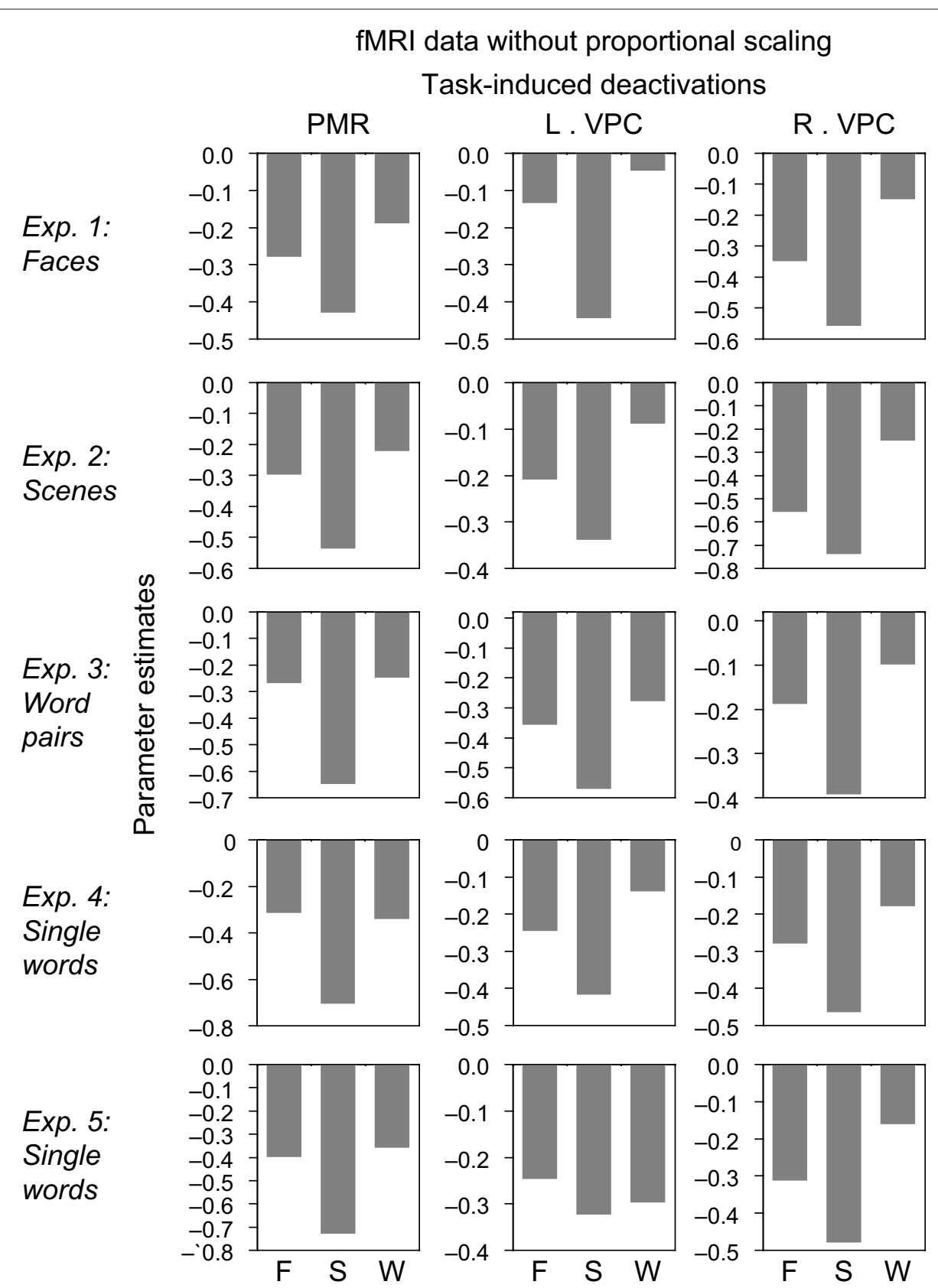

FIGURE 3 |Task-induced deactivations. The PMR and VPC regions identified in Exp. 1-5 showed reliable task-induced deactivation during Blocked Task 1 (faces - F), Blocked Task 2 (scenes - S), and Blocked Task 3 (words -W). Bar graphs indicate mean cluster activity relative to the passive resting baseline.

Table 2 | P values for task-induced deactivations in blocked fMRI tasks 1 (Faces), 2 (Scenes), and 3 (Words).

\begin{tabular}{|c|c|c|c|c|c|c|c|c|c|}
\hline & \multicolumn{3}{|c|}{ PMC } & \multicolumn{3}{|c|}{ L. PLPC } & \multicolumn{3}{|c|}{ R. PLPC } \\
\hline & Faces (F) & Scenes (S) & Words (W) & Faces (F) & Scenes (S) & Words (W) & Faces (F) & Scenes (S) & Words (W) \\
\hline Exp. 1 & 0.004 & 0.001 & $<0.0001$ & 0.054 & 0.005 & 0.218 & $<0.0001$ & $<0.0001$ & $<0.0001$ \\
\hline Exp. 2 & 0.007 & $<0.0001$ & $<0.0001$ & 0.011 & $<0.001$ & 0.035 & $<0.0001$ & $<0.0001$ & 0.001 \\
\hline Exp. 3 & $<0.0001$ & $<0.0001$ & $<0.0001$ & 0.031 & 0.004 & 0.030 & 0.007 & $<0.0001$ & $<0.0001$ \\
\hline Exp. 4 & 0.003 & 0.001 & $<0.0001$ & 0.002 & $<0.0001$ & 0.017 & 0.001 & $<0.0001$ & $<0.0001$ \\
\hline Exp. 5 & 0.011 & 0.006 & 0.010 & $<0.0001$ & $<0.0001$ & 0.000 & 0.003 & $<0.0001$ & $<0.0001$ \\
\hline
\end{tabular}


are part of a default network that is normally deactivated during efficient cognitive performance (McKiernan et al., 2003), we proposed that this effect represents an efficient memory mechanism by which normal default processes are suppressed to allow successful encoding (Daselaar et al., 2004a). Yet, as discussed below, another account suggests that the encoding/retrieval flip reflects the capture of bottom-up attention (Cabeza et al., 2008).

The finding that PMR and VPC consistently show more activity for items that are successfully retrieved (retrieval hits) than for those that are forgotten (retrieval misses) is in line with the notion that episodic retrieval constitutes a prominent part of the default mode (Buckner et al., 2008; Vincent et al., 2006). This idea is supported by several pieces of evidence. First, studies based on verbal reports have indicated that retrieval of episodic memories is an important part of the mental activities occurring during conscious rest (Mazoyer et al., 2001). In addition, populations that show a decline in episodic memory function, such as patients with dementia of the Alzheimer type, also show disruptions in functional activity (Greicius et al., 2004; Petrella et al., 2007; Rombouts et al., 2005) and metabolism (Nestor et al., 2004) in the regions that make up the default network, including PMR and VPC. Third, a recent correlational study based on fMRI data showed that the default regions, including PMR and VPC, are functionally connected to the MTL memory system (Vincent et al., 2006). Finally, cross-experiment comparisons suggest that the regions that support successful retrieval (Henson et al., 2005; Prince et al., 2005; Weis et al., 2004) correspond to the regions that commonly show deactivations during active task performance (Mazoyer et al., 2001; McKiernan et al., 2003; Shulman et al., 1997). The present study provides further support for the role of default network regions in episodic retrieval by showing functional overlap between retrieval success regions and the default network within-participants in five different experiments and four different studies.

In addition to replicating and extending available evidence linking default network regions to episodic memory, the present study provides the first direct evidence that these regions play a very different role during episodic encoding and episodic retrieval. Within the same subjects and the same experiments, we found that the regions that showed increased activation during successful retrieval, showed reduced activation during the encoding of faces, scenes, word pairs, and single words. Thus, together with these previous lines of evidence, the current findings indicate that episodic retrieval is part of the default mode of the brain, whereas episodic encoding is not, and actually benefits from its suppression.

\section{OPPOSING EFFECTS ON ENCODING VS. RETRIEVAL}

The opposite pattern of successful encoding and retrieval activations observed in the present study complements several lines of functional neuroimaging evidence indicating encoding/retrieval dissociations. A first line of evidence concerns the lateralization of prefrontal cortex (PFC) activity. According to the Hemispheric Encoding/Retrieval Asymmetry (HERA) model, encoding is associated more with left, and retrieval more with right, PFC activity (Tulving et al., 1994). This model is primarily supported by evidence from early PET studies that used blocked designs. Interestingly, more recent event-related fMRI studies have not found clear frontal asymmetry differences during encoding and retrieval (Berryhill et al., 2007). This discrepancy might relate to differences between sustained (block design) and transient (event-related design) encoding and retrieval activations. A second encoding/retrieval difference involves the medial temporal lobe memory (MTL) system. According to the Hippocampal Encoding/ Retrieval (HIPER) model, encoding is associated more with anterior, and retrieval more with posterior, MTL activations (Lepage et al., 1998). Though recent event-related fMRI studies provided support for HIPER in relation to successful encoding and retrieval (Daselaar et al., 2004b; Prince et al., 2005), a large meta-analysis of encoding and retrieval studies provided mixed evidence for an antero-posterior gradient in MTL (Schacter and Wagner, 1999). In sum, previously reported dissociations between encoding and retrieval activations have been somewhat inconsistent across studies and appear to be dependent on specific experimental factors. Particularly in PMR that showed perfect spatial overlap across all five experiments, the encoding/retrieval difference in the present study appears to be relatively insensitive to differences in task characteristics. At the same time, it should be noted that VPC did not show this perfect overlap, which may relate to stimulus-related lateralization differences.

In addition to the default mode hypothesis, the dissociation between successful encoding and retrieval activations in PMR and VPC may also have implications for current memory theories. According to a leading theory, successful retrieval of memories requires the reactivation of the same regions involved in the initial encoding of the memories (Alvarez and Squire, 1994; McClelland et al., 1995). This theory is consistent with one of the most important memory principles, the transfer appropriate processing principle, which states that memory is a function of the overlap between encoding and retrieval processes (Morris et al., 1977). This idea is supported by behavioral evidence that minimizing differences between study-test conditions improves memory performance, and by functional neuroimaging findings showing recapitulation of encoding activity in sensory or stimulus-specific regions during retrieval (Hayes et al., 2007; Kahn et al., 2004; Nyberg et al., 2000; Prince et al., 2005; Wheeler et al., 2000). The present findings suggest an exception to these broad theories and general principles. In contrast with the reactivation theory, retrieval activity was found in regions that, instead of being activated during encoding, were actually deactivated. In contrast with the transfer appropriate processing principle, successful memory was associated with an opposing relationship between encoding and retrieval. In fact, the encoding/ retrieval flip leads to the prediction that one or both memory operations should be considerably impaired when the other is performed concurrently. We confirmed this prediction in a recent fMRI study that showed that, when encoding and retrieval are forced to occur within a short period of time, this results in a memory competition with both behavioral and neural costs (Huijbers et al., 2009). It is important to note, however, that the present finding do not invalidate the reactivation theory or the transfer appropriate processing principle; both ideas can be true in general even if there are some exceptions. At the same time, our finding underscores the need for further research on regions showing opposing relationships between encoding and retrieval, and the interactions between these regions and regions supporting recapitulation and transfer appropriate processing. 


\section{ROLE OF VPC IN EPISODIC RETRIEVAL}

A final implication of the encoding/retrieval flip concerns the role of VPC in episodic retrieval.VPC is associated with retrieval success in many functional neuroimaging studies (Cabeza et al., 2008; Vilberg and Rugg, 2008) but damage to this region does not produce severe memory deficits. Recent studies have demonstrated subtle deficits in open-ended episodic memory tasks, such as spontaneous recall of autobiographical events (Berryhill et al., 2007) or the introspective evaluation of recovered memories (Davidson et al., 2008). These subtle deficits suggest that the role of VPC in episodic retrieval might be related to attention, which is one of the main functions attributed to this region (Cabeza, 2008; Mesulam, 1981). An attentional account could also explain the encoding/retrieval flip. Here, we consider two alternative attentional accounts for the encoding-retrieval flip in VPC, the attention to memory (AtoM) account (Cabeza, 2008), and the orienting of internal attention account.

Regarding the AtoM account, we recently proposed that VPC is involved in the capture of reflexive, bottom-up, attention as opposed to voluntary, top-down, attention (Cabeza et al., 2008). The VPC region showing the encoding/retrieval flip in the present study is close to the temporoparietal junction (TPJ) that tends to be deactivated during voluntary attention towards targets. At the same time, TPJ is activated by stimulus-driven reflexive attention to behaviorally relevant stimuli outside the immediate focus of attention (Corbetta and Shulman, 2002; Shulman et al., 2007). The AtoM model holds that for typical encoding tasks, in which the items are the focus of top-down attention and are processed via simple decision judgments, successful encoding critically depends on voluntary attention towards the study items leading to deactivation in TPJ. Yet, when retrieval is successful, voluntary attention towards the memory cue is redirected to the internal mnemonic associations evoked by the memory cue, leading to increased activity in TPJ (Cabeza, 2008). The AtoM account can also explain why TPJ is less active during unsuccessful retrieval, given that retrieval misses do not trigger reflexive attention toward evoked memory associations.

Regarding the orienting of internal attention account, an influential view holds that the default mode regions including VPC are activated during conditions involving internally-oriented attention (Buckner et al., 2008). The default network is active during a variety of internally-oriented task conditions, including thinking about the future (Szpunar et al., 2007), self-referential processing (Kelley et al., 2002), and visual imagery (Hassabis et al., 2007). At the same time, this network is deactivated during demanding tasks requiring externally-oriented attention (McKiernan et al., 2003). Extending these findings to the encoding/retrieval flip in VPC, the orienting

\section{REFERENCES}

Alvarez,P., and Squire,L.R.(1994).Memory consolidation and the medial temporal lobe: a simple network model. Proc. Natl. Acad. Sci. U.S.A. 91, 7041-7045.

Berryhill, M. E., Phuong, L., Picasso, L., Cabeza, R., and Olson, I. R. (2007). Parietal lobe and episodic memory: bilateral damage causes impaired free recall of autobiographical memory. J. Neurosci. 27, 14415-14423.

Buckner, R. L., Andrews-Hanna, J. R., and Schacter, D. L. (2008). The brain's default network: anatomy, function, and relevance to disease. Ann. N. Y. Acad. Sci. 1124, 1-38.

Cabeza, R. (2008). Role of parietal regions in episodic memory retrieval: the dual attentional processes hypothesis. Neuropsychologia 46, 1813-1827.

Cabeza, R., Ciaramelli, E., Olson, I. R., and Moscovitch, M. (2008). The parietal cortex and episodic memory: an attentional account. Nat. Rev. Neurosci. 9, 613-625.

to internal attention account holds that, during successful retrieval, VPC shows enhanced activity involving the orienting of attention to hippocampally-generated internal representations, but during successful encoding, which requires external attention, VPC shows decreased activity involving the efficient suppression of irrelevant internally-generated thoughts. It should be noted, though, that the AToM and orienting to internal attention accounts are not necessarily incompatible. In fact, according to AToM, VPC deactivations are due to top-down attention and VPC activations to bottom-up attention regardless of whether stimuli are internally-generated or externally-presented.

\section{CONCLUSION}

In five different experimental conditions with different stimulus materials, across four separate fMRI studies with unique participants, we found an encoding/retrieval flip in PMR and VPC. During encoding, PMR and VPC showed reduced activity for subsequent hits relative to subsequent misses, whereas during retrieval, they showed increased activity for hits relative to misses. These findings have some implications regarding the default network, the relationship between encoding and retrieval processes, and the role of PMR and VPC in episodic retrieval. First, given that PMR and VPC constitute components of the default network, the opposite levels of activity during successful encoding and retrieval indicate that episodic retrieval processing constitutes part of the default mode activity in the brain, but that encoding processes are not. Second, the opposing relationship between encoding and retrieval represents an exception for accepted memory theories, including the reactivation theory and the transfer-appropriate processing principle. Finally, the current results shed light on the differential role of VPC in episodic encoding and retrieval, which may reflect either differences in voluntary vs. bottom-up attention or internally-oriented vs. externally-oriented attention. More generally, the fact that we found similar results - particularly in PMR - in five different fMRI experiments indicates that the encoding/retrieval flip is reliable and insensitive to specific task requirements.

\section{ACKNOWLEDGEMENTS}

SMD was supported by an NWO VENI grant and the Amsterdam Brain Imaging Platform, NAD by NIA grant T32 AG000029, and RC by NIH grants AG23770 and AG19731. We thank James Kragel for technical assistance.

\section{SUPPLEMENTARY MATERIAL}

Supplementary material can be found online at http:// www.frontiersin.org/humanneuroscience/paper/10.3389/ neuro.09/013.2009/

Corbetta, M., and Shulman, G. L. (2002). Control of goal-directed and stimulusdriven attention in the brain. Nat. Rev Neurosci. 3, 201-215.

Daselaar, S. M., Prince, S. E., and Cabeza, R. (2004a). When less means more: deactivations during encoding that predict subsequent memory. Neuroimage 23, 921-927.

Daselaar, S. M., Veltman, D. J., and Witter, M. P. (2004b). Common pathway in the medial temporal lobe for storage and recovery of words as revealed by event-related functional MRI. Hippocampus 14, 163-169.

Davidson, P. S., Anaki, D., Ciaramelli, E., Cohn, M., Kim, A. S., Murphy, K. J., Troyer, A. K., Moscovitch, M., and Levine, B. (2008). Does lateral parietal cortex support episodic memory? Evidence from focal lesion patients. Neuropsychologia 46, 1743-1755.

Greicius, M.D., Srivastava, G., Reiss, A. L., and Menon, V. (2004). Default-mode network activity distinguishes Alzheimer's disease from healthy 
aging: evidence from functional MRI. Proc. Natl. Acad. Sci. U.S.A. 101, 4637-4642.

Gusnard, D. A., and Raichle, M. E. (2001). Searching for a baseline: functional imaging and the resting human brain. Nat. Rev. Neurosci. 2, 685-694.

Hassabis, D., Kumaran, D., and Maguire, E. A. (2007). Using imagination to understand the neural basis of episodic memory. J. Neurosci. 27, 14365-14374.

Hayes, S. M., Nadel, L., and Ryan, L. (2007). The effect of scene context on episodic object recognition: parahippocampal cortex mediates memory encoding and retrieval success. Hippocampus $17,873-889$.

Henson, R. N., Hornberger, M., and Rugg, M.D. (2005). Further dissociating the processes involved in recognition memory: an FMRI study. J. Cogn. Neurosci. 17, 1058-1073.

Huijbers, W., Pennartz, C.M., Cabeza, R., and Daselaar, S. M. (2009). When learning and remembering compete: a functional MRI study. PLoS Biol 7, ell.

Kahn, I., Davachi, L., and Wagner, A. D. (2004). Functional-neuroanatomic correlates of recollection: implications for models of recognition memory. J. Neurosci. 24, 4172-4180.

Kao, Y. C., Davis, E. S., and Gabrieli, J. D. (2005). Neural correlates of actual and predicted memory formation. Nat. Neurosci. 8, 1776-1783.

Kelley, W. M., Macrae, C. N., Wyland, C. L., Caglar, S., Inati, S., and Heatherton, T. F. (2002). Finding the self? An event-related fMRI study. J. Cogn. Neurosci, 14, 785-794.

Kennedy, D. P., Redcay, E., and Courchesne, E. (2006). Failing to deactivate: resting functional abnormalities in autism. Proc. Natl. Acad. Sci. U.S.A. 103, 8275-8280.

Lepage, M., Habib, R., and Tulving, E. (1998). Hippocampal PET activations of memory encoding and retrieval: the hiper model. Hippocampus 8 , 313-322.

Liang, M., Zhou, Y., Jiang, T., Liu, Z., Tian, L., Liu, H., and Hao, Y. (2006). Widespread functional disconnectivity in schizophrenia with resting-state functional magnetic resonance imaging. Neuroreport 17, 209-213.

Liu, H., Liu, Z., Liang, M., Hao, Y., Tan, L., Kuang, F., Yi, Y., Xu, L., and Jiang, T. (2006). Decreased regional homogeneity in schizophrenia: a resting state functional magnetic resonance imaging study. Neuroreport 17, 19-22.

Lustig, C., Snyder, A. Z., Bhakta, M., O’Brien, K. C., McAvoy, M., Raichle, M. E., Morris, J. C., and Buckner, R. L.
(2003). Functional deactivations: change with age and dementia of the Alzheimer type. Proc. Natl. Acad. Sci. U.S.A. 100, 14504-14509.

Mazoyer, B., Zago, L., Mellet, E., Bricogne, S., Etard, O., Houde, O., Crivello, F., Joliot, M., Petit, L., and Tzourio-Mazoyer, N. (2001). Cortical networks for working memory and executive functions sustain the conscious resting state in man. Brain Res. Bull. 54, 287-298.

McClelland, J. L., McNaughton, B. L., and O’Reilly, R. C. (1995). Why there are complementary learning systems in the hippocampus and neocortex: insights from the successes and failures of connectionist models of learning and memory. Psychol. Rev. 102, 419-457.

McKiernan, K. A., Kaufman, J. N., KuceraThompson, J., and Binder, J. R. (2003). A parametric manipulation of factors affecting task-induced deactivation in functional neuroimaging. J. Cogn . Neurosci. 15, 394-408.

Mesulam,M.M.(1981).A cortical network for directed attention and unilateral neglect. Ann. Neurol. 10, 309-325.

Miller, S. L., Celone, K., DePeau, K., Diamond,E.,Dickerson, B.C.,Rentz, D., Pihlajamaki, M., and Sperling, R. A. (2008). Age-related memory impairment associated with loss of parietal deactivation but preserved hippocampal activation. Proc. Natl. Acad. Sci. U.S.A. 105, 2181-2186.

Minear, M. D., and Park, C. (2004). A lifespan database of adult facial stimuli. Behav Res Methods Instrum Comput. 36, 630-633.

Morris, C. D., Bransford, J. D., and Franks, J.J. (1977).Levels of processing versus transfer appropriate processing. J Verbal Learn Verbal Behav 16, 519-533.

Nestor, P. J., Scheltens, P., and Hodges, J. R. (2004). Advances in the early detection of Alzheimer's disease. Nat. Med. 10(Suppl), S34-S41.

Nyberg, L., Habib, R., Mcintosh, A., and Tulving, R. E. (2000). Reactivation of encoding-related brain activity during memory retrieval. Proc. Natl. Acad. Sci. U.S.A. 97, 11120-11124.

Otten, L. J., Quayle, A. H., Akram, S., Ditewig, T.A., and Rugg, M. D. (2006). Brain activity before an event predicts later recollection. Nat. Neurosci. 9, 489-491.

Otten, L. J., and Rugg, M. D. (2001). When more means less: neural activity related to unsuccessful memory encoding. Curr. Biol. 11, 1528-1530.

Petrella, J. R., Prince, S. E., Wang, L., Hellegers, C., and Doraiswamy, P. M. (2007). Prognostic value of posteromedial cortex deactivation in mild cognitive impairment. PLOS ONE 2, el104.

Prince, S. E., Daselaar, S. M., and Cabeza, R. (2005). Neural correlates of relational memory: successful encoding and retrieval of semantic and perceptual associations. J. Neurosci. 25, 1203-1210.

Prince, S. E., Dennis, N.A., and Cabeza, R. (2009). Encoding and retrieving faces and places: distinguishing processand stimulus-specific differences in brain activity. Neuropsychologia 47 , 2282-2289.

Prince, S. E., Tsukiura, T., and Cabeza, R. (2007). Distinguishing the neural correlates of episodic memory encoding and semantic memory retrieval. Psychol. Sci. 18, 144-151.

Raichle,M.E.,MacLeod,A.M.,Snyder, A. Z., Powers, W. J., Gusnard, D. A., and Shulman, G. L. (2001). A default mode of brain function. Proc. Natl. Acad. Sci. U.S.A. 98, 676-682.

Rombouts, S. A., Barkhof, F., Goekoop, R., Stam, C. J., and Scheltens, P. (2005) Altered resting state networks in mild cognitive impairment and mild Alzheimer's disease: an fMRI study. Hum. Brain Mapp. 26, 231-239.

Schacter, D. L., and Wagner, A. D. (1999). Medial temporal lobe activations in fMRI and PET studies of episodic encoding and retrieval. Hippocampus, 9, 7-24.

Schon, K., Hasselmo, M.E.,Lopresti, M. L., Tricarico, M.D., and Stern,C.E. (2004) Persistence of parahippocampal representation in the absence of stimulus input enhances long-term encoding: a functional magnetic resonance imaging study of subsequent memory after a delayed match-to-sample task. J Neurosci. 24, 11088-11097.

Shrager, Y., Kirwan, C. B., and Squire, L. R. (2008). Activity in both hippocampus and perirhinal cortex predicts the memory strength of subsequently remembered information. Neuron $59,547-553$.

Shulman, G.L.,Astafiev,S.V.,McAvoy, M. P., d'Avossa, G., and Corbetta, M. (2007) Right TPJ deactivation during visual search: functional significance and support for a filter hypothesis. Cereb. Cortex. 17, 2625-2633.

Shulman, G. L., Fiez, J. A., Corbetta, M. Buckner, R. L., Miezin, F. M. Raichle, M. E., and Petersen, S. E. (1997). Common blood flow changes across visual tasks: 3 . Decreases in cerebral cortex. Journal of Cognitive Neuroscience 9, 648-663.

Szpunar, K. K., Watson, J. M., and McDermott, K. B. (2007). Neural substrates of envisioning the future. Proc. Natl. Acad. Sci. U.S.A. 104, 642-647.
Talairach, J., and Tournoux, P. (1988). Co-Planar Stereotaxic Atlas of the Human Brain. Stuttgart, Thieme Verlag.

Tulving, E., Kapur, S., Craik, F. I., Moscovitch, M., and Houle, S. (1994). Hemispheric encoding/retrieval asymmetry in episodic memory: positron emission tomography findings. Proc. Natl. Acad. Sci. U.S.A. 91, 2016-2020

Vilberg, K. L., and Rugg, M. D. (2008). Memory retrieval and the parietal cortex: a review of evidence from a dualprocess perspective. Neuropsychologia 46, 1787-1799.

Vincent, J. L., Snyder, A. Z., Fox, M. D., Shannon, B. J., Andrews, J. R. Raichle, M. E., and Buckner, R. L. (2006). Coherent spontaneous activity identifies a hippocampal-parietal memory network. J. Neurophysiol. 96, 3517-3531.

Wagner, A. D., and Davachi, L. (2001) Cognitive neuroscience: forgetting of things past. Curr. Biol. 11, R964-R967.

Wagner,A.D., Shannon, B.J., Kahn, I., and Buckner, R. L. (2005). Parietal lobe contributions to episodic memory retrieval. Trends Cogn. Sci. 9, 445-453.

Weis, S., Klaver, P., Reul, J., Elger, C. E., and Fernandez, G. (2004). Temporal and cerebellar brain regions that support both declarative memory formation and retrieval. Cereb. Cortex 14, 256-267.

Wheeler, M. E., Petersen, S. E., and Buckner, R. L. (2000). Memory's echo: vivid remembering reactivates sensory-specific cortex. Proc. Natl. Acad. Sci. U.S.A. 20, 11125-11129.

Conflict of Interest Statement: The authors declare that the research was conducted in the absence of any commercial or financial relationships that could be construed as a potential conflict of interest.

Received: 22 April 2009; paper pending published: 14 May 2009; accepted: 22 June 2009; published online: 20 July 2009.

Citation: Daselaar SM, Prince SE, Dennis NA, Hayes SM, Kim H and Cabeza R (2009) Posterior midline and ventral parietal activity is associated with retrieval success and encoding failure. Front. Hum. Neurosci. (2009) 3:13. doi: 10.3389/neuro.09.013.2009

Copyright $\odot 2009$ Daselaar, Prince, Dennis, Hayes, Kim and Cabeza. This is an openaccess article subject to an exclusive license agreement between the authors and the Frontiers Research Foundation, which permits unrestricted use, distribution, and reproduction in any medium, provided the original authors and source are credited. 